\title{
Salvage Esophagectomy for Residual Tumor After Definitive Chemoradiotherapy for Esophageal Squamous Cell Carcinoma Invading the Neighboring Organs: Is it a Feasible Choice?
}

\author{
Masayuki Watanabe, MD, PhD, FACS \\ Department of Gastroenterological Surgery, Cancer Institute Hospital of Japanese Foundation for Cancer Research, Tokyo, \\ Japan
}

Treatment of locally advanced esophageal cancer invading the neighboring organs is challenging. Among the two major histologic subtypes, adenocarcinoma, most often located in the lower esophagus, can invade the descending aorta, lung, and pericardium. Meanwhile, squamous cell carcinoma (SCC), located predominantly in the middle and upper esophagus, is more likely to invade the trachea, bronchus, and aortic arch. For patients who present with extension into such vital organs, definitive chemoradiotherapy (dCRT) is indicated as the standard choice. For those with SCC, a complete response (CR) is often achieved, even among those with initially unresectable disease. This leads to the ongoing discussion of whether there is room for surgical treatment of residual tumor after dCRT.

Booka et al. reported their experience of salvage esophagectomy for patients with initially unresectable, locally advanced T4 esophageal SCC. ${ }^{1}$ Among 317 patients who underwent dCRT in this situation, $71(22.4 \%)$ achieved a CR, while $18(5.7 \%)$ of 246 patients did not respond to dCRT experienced salvage esophagectomy. Among those patients who did not respond to dCRT, the long-term survival of patients with salvage esophagectomy was significantly better than those without. R0 resection was the only independent prognostic factor in patients with salvage esophagectomy, and all incomplete resections

(C) Society of Surgical Oncology 2020

First Received: 20 April 2020;

Published Online: 7 May 2020

M. Watanabe, MD, PhD, FACS

e-mail: masayuki.watanabe@jfcr.or.jp occurred when the tumors were located in the upper esophagus. No mortality was observed, and the 5-year overall survival of the salvage esophagectomy group was $51.6 \%$. I express my sincere respect for excellent outcomes.

Several authors have reported the results of salvage esophagectomy after dCRT for initially unresectable T4 esophageal SCC. The R0 resection rates differed significantly among the studies, ranging from 39.2 to $92.3 \%$, while the operative mortality rates also ranged from 0 to $10.2 \% .^{1-5}$ These differences are mainly due to the differences in the patient selection criteria for salvage surgery among the institutes, because all the studies were only conducted in high-volume esophageal cancer centers. The results indicate the difficulty in appropriate patient selection in this particular situation. Meanwhile, R0 resection is commonly demonstrated as a prognostic factor, although the survival outcomes also differed among the studies.

There are several problems in the diagnosis of locally advanced, unresectable esophageal SCC. First, an accurate diagnosis of 'initially unresectable' remains challenging despite the progress in the imaging modalities. Although the gold standard for the determination of T4 esophageal cancer is computed tomography (CT), it is difficult to differentiate between tumors with expanding growth and those with invasive growth. Endoscopic ultrasonography is also useful to diagnose the depth of tumor invasion, but accurate scanning is often tricky due to esophageal stenosis in cases with T4 tumors. Second, the diagnosis of CR is often inaccurate. In the study by Booka et al., 5 of 18 patients $(27.8 \%)$ who underwent salvage esophagectomy had complete pathologic responses. ${ }^{1}$ As the long-term outcomes of patients who achieved CR were favorable, salvage esophagectomy may not be necessary for these 
patients. Recently, the preSANO trial revealed that clinical evaluation with endoscopic ultrasonography, bite-on-bite biopsies, and fine-needle aspiration of suspicious lymph nodes was adequate for the detection of locoregional residual disease, with positron emission tomography (PET)/CT for the detection of interval metastases after neoadjuvant CRT. ${ }^{6}$ The diagnostic accuracy of CR after dCRT can be improved by using a combination of these modalities.

$\mathrm{R} 0$ resection is mandatory to achieve long-term survival after salvage esophagectomy. As Booka et al. reported, the upper esophageal location of the tumors was a risk factor for incomplete resection, suggesting that airway invasion is a significant cause of incomplete resection. ${ }^{1}$ Although an extensive operation with combined resection of the major airway for esophageal cancer invading the tracheobronchial tree has been reported by some investigators, ${ }^{7}$ the efficacy and safety have not yet been elucidated. Recently, acceptable short-term outcomes of salvage esophagectomy combined with partial aortic wall resection after thoracic endovascular aortic repair have been reported. ${ }^{8}$ Further investigations are required to evaluate the efficacy and safety of such extended procedures for salvage esophagectomy.

Recently, a multicenter, phase II trial assessing the safety and effectiveness of chemoselection with induction chemotherapy using docetaxel plus cisplatin and 5-fluorouracil, and subsequent conversion surgery for initially unresectable locally advanced esophageal SCC, was conducted in Japan. ${ }^{9}$ Twenty of 48 patients enrolled (41.7\%) underwent conversion surgery, with 19 (39.6\%) achieving $\mathrm{R} 0$ resection. The 3-year overall and progression-free survival rates of patients who achieved R0 resection were $71.4 \%$ and $61.3 \%$, respectively. ${ }^{10}$ These results indicate that chemoselection, where induction therapy is administered and patients with demonstrated treatment response then undergo resection, is an alternative strategy for the treatment of initially unresectable, locally advanced SCC. A phase III trial comparing this strategy versus dCRT plus salvage surgery is ongoing. ${ }^{11}$

In summary, in this series of selected patients with residual tumors after dCRT for locally advanced T4 esophageal SCC, salvage esophagectomy provided long-term survival when R0 resection was achieved. This study highlights that further improvement of the pretreatment diagnosis of $\mathrm{T} 4$ invasion and accurate evaluation of the treatment effect are needed. In addition, better assessment of the efficacy of the chemoselection strategy is required to improve outcomes in this challenging patient cohort.

DISCLOSURE Masayuki Watanabe has no disclosures to declare.

\section{REFERENCES}

1. Booka E, Haneda R, Ishii K, et al. Appropriate candidates for salvage esophagectomy of initially unresectable, locally advanced T4 esophageal squamous cell carcinoma. Ann Surg Oncol. 2020. https://doi.org/10.1245/s10434-020-08440-7.

2. Ikeda K, Ishida K, Sato N, et al. Chemoradiotherapy followed by surgery for thoracic esophageal cancer potentially or actually involving adjacent organs. Dis Esophagus. 2001;14:197-201.

3. de Manzoni G, Pedrazzani C, Pasini F, et al. Chemoradiotherapy followed by surgery for squamous cell carcinoma of the thoracic esophagus with clinical evidence of adjacent organ invasion. $J$ Surg Oncol. 2007;95:261-6.

4. Ohkura Y, Ueno M, Iizuka T, Udagawa H. Prognostic Factors and Appropriate Lymph Node Dissection in Salvage Esophagectomy for Locally Advanced T4 Esophageal Cancer. Ann Surg Oncol. 2019;26:209-16.

5. Okamura A, Hayami M, Kozuki R, et al. Salvage esophagectomy for initially unresectable locally advanced T4 esophageal squamous cell carcinoma. Esophagus. 2020;17:59-66.

6. Noordman BJ, Apaander MCW, Valkema R, et al. Detection of residual disease after neoadjuvant chemoradiotherapy for oesophageal cancer (preSANO): a prospective multicentre, diagnostic cohort study. Lancet Oncol. 2018;19:965-74.

7. Ferri L. Clinical T4b esophageal cancer: can we make an 'unresectable' tumours resectable? Ann Surg Oncol. 2020;27:329-30.

8. Nakajima M, Muroi H, Kikuchi M, et al. Salvage esophagectomy combined with partial aortic wall resection following thoracic endovascular aortic repair. Gen Thorac Cardiovasc Surg. 2018;66:736-43.

9. Yokota T, Kato K, Hamamoto Y, et al. Phase II study of chemoselection with docetaxel plus cisplatin and 5-fluorouracil induction chemotherapy and subsequent conversion surgery for locally advanced unresectable oesophageal cancer. Br J Cancer. 2016;115:1328-34.

10. Yokota T, Kato K, Hamamoto Y, et al. A 3-year overall survival update from a phase 2 study of chemoselection with DCF and subsequent conversion surgery for locally advanced unresectable esophageal cancer. Ann Surg Oncol. 2020;27:460-7.

11. Terada M, Hara H, Daiko H, et al. Phase III study of tri-modality combination therapy with induction docetaxel plus cisplatin and 5-fluorouracil versus definitive chemoradiotherapy for locally advanced unresectable squamous-cell carcinoma of the thoracic esophagus (JCOG1510: TRIANgLE). Jpn J Clin Oncol. 2019;49:1055-60.

Publisher's Note Springer Nature remains neutral with regard to jurisdictional claims in published maps and institutional affiliations. 